\title{
Anisotropic Models of Dark Halos
}

\author{
N. Ya. Sotnikova and S. A. Rodionov \\ Sobolev Astronomical Institute, \\ St. Petersburg State University, \\ Universitetskii pr. 28, 198904 Russia
}

\begin{abstract}
An iterative approach is used to construct spherically symmetric equilibrium models with an anisotropic velocity distribution. The potentialities of the method have been tested on models with known distribution functions, the Osipkov-Merritt models. It is shown that models that differ significantly from the Osipkov-Merritt models can be constructed. An $N$-body model of a dark halo with a density distribution that approximates the results of cosmological simulations (the Navarro-Frenk-White model) has been constructed. The anisotropy profile has been taken to be similar to that yielded by cosmological simulations. The constructed models can serve as direct input data for investigating the dynamics and stability of such systems in $N$-body simulations.
\end{abstract}

${ }^{1}$ e-mail: nsot@astro.spbu.ru

${ }^{2}$ e-mail: seger@astro.spbu.ru 


\section{INTRODUCTION}

As a rule, several gravitationally significant subsystems that differ in structure and dynamics are identified in galaxies. Spiral galaxies are the most complex in this respect. They consist of a disk, a bulge, a compact nucleus, and an extended dark halo. Multicomponent models are required to properly describe their dynamics. The calculations of the dynamical evolution of composite self-consistent galactic models, including a massive dark halo, are known to be very time-consuming. In the 1980s and 1990s, numerical simulations with interacting galaxies (beginning with the paper by Barnes (1988)) were almost the only area of research in which the computational costs were justified. The introduction of a live halo instead of a rigid one specified analytically into the models changed radically the outcome of the interaction. The galaxies rapidly merged together during close collisions. This was the result of the action of dynamical friction due to the presence of massive dark halos.

As regards the models of isolated galaxies (both disk and elliptical ones), a "cheaper" approach had long been applied here. Usually, the dark halo potential was assumed to be rigidly fixed. Moreover, using a live halo at a moderately large number of particles $N$ led to inadequate dynamical evolution of the model galaxies. The stellar disk was rapidly heated in the vertical direction (Walker et al. 1996). All of the collective processes that gave rise, for instance, to transient spirals and bars were also accelerated in the disks. Thus, for example, Walker et al. (1996) provided the growth rate of the bar-mode amplitude as a function of the number of halo-modeling particles. In models with a rigid halo, the bar mode did not manifest itself on time scales of 3 Gyr. A similar behavior of the model was observed only when a very large number of particles in the halo $(N=450000)$ was used.

The potentialities of the modern computer technology have gradually led to mass simulations with a large number of particles and, hence, with a live halo. However, the realization of the importance of taking into account the change in the angular momentum of disk stars during the interaction with resonant particles not only in the disk, but also in the live halo was more significant. Thus, for example, the proper description of the mentioned exchange processes prompted a revision of the views about disk stabilization with respect to the bar mode and about the direction of evolution of the bars in disk galaxies with a dark halo (Athanassoula 2003) that had prevailed for thirty years (since the paper by Ostriker and Peebles (1973)).

Cosmological simulations in which the formation of galaxies is modeled are another area of application of self-consistent models with a dark halo. The formation of disk galaxies is currently believed to be related to gas accretion onto dark halos. The dark halos themselves result from hierarchical clustering of dark matter density fluctuations (for the first $N$-body models of this process, see Efstathiou and Jones 1979).

For many dynamical problems, it is important to have simple algorithms for constructing equilibrium models of a live halo. By this we mean the distribution of $\mathrm{N}$ particles representing the system in phase space. A live equilibrium dynamical model is fairly easy to specify if spherical symmetry and an isotropic particle velocity distribution are assumed. The most fruitful approach is to use a distribution function (DF) in the form $f(E)$, where $E$ is the specific energy. According to the Jeans theorem, this ensures that an equilibrium gravitating model will be obtained. If the DF is unknown, but there are considerations regarding the density profile $\rho(r)$, then $f(E)$ can be determined (analytically or numerically) via Abel's integral relation, which is often called Eddington's formula (see, e.g., Binney and Tremaine 1987). Thus, for example, for the dark halo models of great interest, the Navarro-Frenk-White model (hereafter the NFW model; Navarro et al. 1996, 1997) 
and the Moore et al. (1999) model (hereafter the M99 model), the corresponding DFs for the isotropic case can be easily obtained numerically (Widrow 2000; Lokas and Mammon 2001). There exist good analytic fits to $f(E)$ (Widrow 2000). Their existence, in fact, completely solves the problem of equilibrium initial conditions for numerical $N$-body simulations when the isotropic NFW and M99 models are used. Another approach is to use the Jeans equations and the assumption that the velocity distribution is Gaussian (Hernquist 1993). However, this approach does not guarantee an equilibrium.

Passing to anisotropic models, even if spherically symmetric ones, slightly complicates the preparation of numerical simulations. In this case, a DF in the form $f(E, L)$, where $L$ is the magnitude of the particle angular momentum vector, should be used to describe an equilibrium (nonrotating) stellar system. The degree of anisotropy of the velocity distribution described by the parameter (Binney and Tremaine 1987) $\beta=1-\frac{\sigma_{\theta}^{2}}{\sigma_{r}^{2}}$, where $\sigma_{\theta}^{2}$ and $\sigma_{r}^{2}$ are the velocity dispersions, respectively, in the $\theta$ and $r$ directions in spherical coordinates ( $\beta=0$ is isotropic case and $\beta=1$ is the case of purely radial orbits). In such models, $\sigma_{\theta}^{2}=\sigma_{\varphi}^{2}$, where $\sigma_{\varphi}^{2}$ is the stellar velocity dispersion in the $\varphi$ direction.

The methods of finding the DF (analytically or numerically) for anisotropic models of a special type, the so-called OsipkovMerritt models (hereafter the OM models; Osipkov 1979; Merritt 1985a, 1985b), are well known. The formalism of Abel's integral transform with a slight modification is also applicable here. Widrow (2000) provided DF fits for the OM models with the NFW and M99 density profiles (and for some other models).

Anisotropic spherically symmetric models can be used to investigate the dynamical evolution of systems with a live halo. The most popular halo models are NFW and M99. These were introduced to fit the universal density profiles of dark halos obtained in numerical calculations within the framework of the modern paradigm of galaxy formation through successive mergers of cold dark matter (CDM) fluctuations. The mergers result in an hierarchy of dark halos that differ in mass by several orders of magnitude. The halos themselves serve as an active background against which all of the scenarios for structure formation and galaxy formation from baryonic matter, gas, unfold (semianalytical models; White and Rees 1978; Fall and Efstathiou 1980; Blumenthal et al. 1986; Mo et al. 1998).

Cosmological $N$-body simulations show that the forming halos far from the center are not described by isotropic models. However, the anisotropy profile $\beta(r)$ is also far from that yielded by the OM models. The generalized models by Cuddeford (1991) are also unsuitable. For them, just as for the OM models, the velocity anisotropy profile on the periphery is produced by purely radial orbits ( $\beta$ tends to 1 ). At the same time, for the dark halos obtained in cosmological simulations, the degree of anisotropy is small far from the center (Cole and Lacey 1996; Thomas et al. 1998; Colin et al. 2000; Fukushige and Makino 2001; Diemand et al. 2004).

Specifying models of anisotropic dark halos compatible with the results of numerical simulations, we can proceed in two ways. For example, we can take models with a small $N$ directly from cosmological simulations and use the technique of increasing the phase density resolution (Klypin et al. 2001) to that required ( $N$ is of the order of several hundred thousand) in simulations with isolated galaxies (e.g., Curir et al. 2007). Alternatively, we can construct $N$-body models with a fixed anisotropy profile for a given density distribution from the outset. In the latter case, one of the two recently suggested methods is suitable. The first method is based on a generalization of the OM and Cuddeford (1991) method. In this method (Baes and Van Hese 2007), a phase density distribution function 
can be derived for models in which $\beta$ does not tend to unity far from the center using a complex analytical technique. The second method is based on an iterative approach to constructing equilibrium dynamical models of galaxies and their subsystems (Rodionov and Sotnikova 2006; Rodionov and Orlov 2008). In this paper, we extend the range of applicability of the suggested iterative method by using it in the context of constructing models of anisotropic spherically symmetric dark halos with a fixed velocity anisotropy profile, thereby expanding the class of equilibrium models with prefixed properties.

\section{THE ITERATIVE METHOD OF CON- STRUCTING EQUILIBRIUM MODELS}

\subsection{The General Idea of the Iterative Method}

The iterative method is designed to construct nearly equilibrium $N$-body models with a fixed density distribution (Rodionov and Sotnikova 2006; Rodionov and Orlov 2008). It does not require any knowledge of an explicit expression for the distribution function (DF) or the profiles of the moments of the velocity distribution. It is only necessary to determine the type of the system and to specify constraints on its kinematics.

The main idea of the iterative method is that we let the system to come to equilibrium itself through its dynamical evolution by holding the needed parameters. The method is implemented using the following algorithm:

1. An $N$-body model with a fixed density distribution but with an arbitrary velocity distribution is constructed. For example, the particle velocities may be taken to be zero.

2. The model is allowed to evolve on a short time scale that is generally shorter than the system crossing time.

3. A model with the same velocity distribution as that in the evolved model but with the initial density profile is constructed, i.e., the density profile is restored at the end of each iteration. This procedure can also be considered as the transfer of the evolving velocity distribution to the specified density model.

If there are initial constraints on the particle velocity distribution, then this distribution is also "corrected" by taking into account the available constraint:1 (for a discussion, see below).

4. Return to item (2).

The iterations are terminated when the velocity distribution ceases to change.

As a result, we obtain an $N$-body model with a fixed density profile and fixed global kinematics that is very close to equilibrium (Rodionov and Sotnikova 2006; Rodionov and Orlov 2008).

In the iterative method, the system reaches equilibrium itself in a fixed potential. Our approach differs from previously suggested approaches, in which the system's selfadjustment to equilibrium is also used (see, e.g., Barnes 1988), in that we forcibly restore the required parameters and profiles to the system at the end of each iteration.

\footnotetext{
${ }^{1}$ Isotropic models for which all velocity vectors should be mixed in directions when the velocity field is transferred (Rodionov and Sotnikova 2006) are the simplest example.
} 
The idea of our iterative method is very simple. However, when it is implemented in practice, difficulties arise at the third stage, the stage of transferring the velocity distribution from the evolved model to the model with the required density profile. Here, we use the velocity distribution transfer algorithm suggested by Rodionov and Orlov (2008).

\subsection{The Velocity Distribution Transfer Algorithm}

The objective of the transfer is as follows. We have the "old" model, a slightly evolved model obtained at the end of the preceding iteration. We wish to copy the velocity distribution from this model. We also have the "new" model constructed with a fixed density distribution. We should assign velocities to the particles in the new model according to the velocity distribution in the old model.

The method for transferring the velocity distribution from the old, evolved model to the new one was described in detail by Rodionov and Orlov (2008). Here, we only briefly remind the "transfer" algorithm that we use.

The main idea of the algorithm is to assign exactly the same velocities to the particles in the new model as the velocities of the particles closest to them in the old model.

The number of neighbors, $n_{n b}$, is an input parameter of the algorithm. For each particle from the old model, we introduce a variable $n_{\mathrm{use}}^{j}$, the number of times this particle is used to copy its velocity. At the beginning of the algorithm, we set $n_{\text {use }}^{j}=0$.

First, we examine all particles in the new model, fix the position of each such particle $\mathbf{r}_{i}$, and find the number of particles $n_{n b}$ from the old model that are closest to the position $\mathbf{r}_{i}$. Next, we separate out a subgroup of particles with the minimum $n_{\text {use }}^{j}$ from this group and determine the particle closest to the position $\mathbf{r}_{i}$ in this subgroup. The velocity of the particle found from the old model is assigned to the particle under consideration from the new model. One is added to the variable $n_{\text {use }}^{j}$ for the particle found from the old model.

For spherically symmetric systems, it will suffice to seek for the nearest neighbors according to the criterion for closeness of the radial coordinate. Thus, all of the particles located in a thin spherical layer into which the particle under consideration falls are considered neighbors here. This technique improves significantly the statistics and ensures that the constructed model will have a spherically symmetric velocity field, i.e., the velocity distribution will depend only on the radial coordinate. In this case, the velocities should be "transferred" in a spherical coordinate system.

\subsection{Constraints on the Velocity Distribution}

In the iterative method, the system makes a transition to equilibrium itself in the course of its independent dynamical evolution. The direction of the transition is not arbitrary. It is specified by forcibly fixing the density distribution and certain kinematic parameters. If only one equilibrium model can exist for these parameters, then one might expect the system to come precisely to this equilibrium state in the process of iterations. Thus, for example, when constructing isotropic spherically symmetric models, we can maintain only the condition for equivalence of the three velocity components without imposing any constraints on the velocity dispersion profiles. The system itself comes to the only possible state that corresponds to the function $f(E)$ for a given density profile (Rodionov and Sotnikova 2006).

If more than one equilibrium model can exist at fixed parameters, then an ambiguity emerges. The question arises as to where the iterative process will lead to in this case. One 
might expect that the result of the iterative method in this case will depend on the initial conditions, while the iterations will converge, in a sense, to the "closest" or distinguished (according to particular criteria) equilibrium state.

The problem of nonuniqueness of the models obtained also exists for the well-known method of Schwarzschild $(1979,1993)$, which uses a library of orbits in a fixed potential. This method was suggested to construct generally triaxial models described via three integrals of motion. No analytical representation is required for these integrals. It will suffice to know only the density distribution and, accordingly, the potential of the system. However, without a predetermined criterion for the selection of orbits in a fixed potential, their superposition gives a whole set of models that correctly represent the require density distribution.

In our method, in constructing spherically symmetric models, an ambiguity can arise when minimal constraints are imposed on the kinematics of the system. Thus, for example, if the mean degree of anisotropy is fixed for the entire model, then a whole set of equilibrium anisotropic models with the same density profile can be obtained, depending on the initial conditions on the velocity distribution. All of the available information about the global kinematics of the system should be used to avoid this ambiguity.

The goal of this paper is to construct equilibrium spherically symmetric models with a fixed (generally arbitrary) anisotropy profile. To this end, in addition to the density profile, we also held the anisotropy profile in the process of iterations. This was achieved in the following way. At the end of each iteration, after a readjustment of the model (with the same velocity distribution as that in the slightly evolved model from the preceding iteration step), we corrected the particle velocities in such a way that the system had the required anisotropy profile. For this purpose, we divided the model into spherical layers. Each layer contained approximately the same number of particles. Next, we calculated the required degree of anisotropy $\beta_{k}$ for each layer based on the fixed anisotropy profile. The velocities of all particles in the layer were changed in such a way that the degree of anisotropy averaged inside the layer was exactly equal to $\beta_{k}$. The velocities can be corrected, for example, as follows:

$$
\begin{aligned}
& v_{\varphi, i}=v_{\varphi, i}^{\prime} \sqrt{1-\beta_{k}} \frac{\sigma_{r}^{\prime}}{\sigma_{\varphi}^{\prime}} \\
& v_{\theta, i}=v_{\theta, i}^{\prime} \sqrt{1-\beta_{k}} \frac{\sigma_{r}^{\prime}}{\sigma_{\theta}^{\prime}},
\end{aligned}
$$

where $\sigma_{r}^{\prime}, \sigma_{\varphi}^{\prime}$ and $\sigma_{\theta}^{\prime}$ are the current particle velocity dispersions in the layer in the $r, \varphi$ and $\theta$ directions, respectively; $v_{\varphi, i}^{\prime}$ and $v_{\theta, i}^{\prime}$ are the current $\varphi$ and $\theta$ velocity components of particle $i ; v_{\varphi, i}$ and $v_{\theta, i}$ are the corrected velocity components.

There is some freedom in choosing a method for correcting the particle velocity in the layer. In the method described above, we corrected only the $\varphi$ and $\theta$ velocity components. All three velocity components can be corrected in such a way that the total change was minimal according to some chosen criterion. Test calculations show that the result is virtually independent of the details of the chosen procedure.

Note also that we were interested in models with equivalent $\varphi$ and $\theta$ velocity components, i.e., we considered models without rotation. To meet this condition when transferring the velocity distribution, we "mixed" these velocity components. 


\section{EQUILIBRIUM ANISOTROPIC SPHERI- CALLY SYMMETRIC MODELS}

Subsequently, we used the TREE algorithm (Barnes and Hut 1986) and some of the codes from the NEMO package (http://astro.udm.edu/nemo; Teuben 1995) to numerically solve the $N$-body problem. All models were constructed for $N=100000$. We chose the integration step $d t$ and the softening length of the gravitational potential $\epsilon$ in our $N$-body simulations according to recommendations from Rodionov and Sotnikova (2005).

The calculations were performed in a virial system of units. In this system of units, the gravitational constant is $G=1$, the total mass of the model is $M=1$, and the total energy of the system is $E=-1 / 4$. In the NFW models, for which the total mass diverges, the system of units used is specified separately.

\section{$3.1 \quad$ Test Models}

Nearly homogeneous models. A Plummer sphere. In stellar dynamics, there are several analytical models that are used to test the efficiency of numerical methods. If the suggested numerical technique completely reproduces the dynamics of known models, then it can also be applied in more complex problems.

In the previous section, we described a realization of the iterative method for constructing equilibrium spherically symmetric models with a fixed density profile and a fixed anisotropy profile. To demonstrate the potentialities of the described method, it is useful to turn to well-known anisotropic models for which there are analytical expressions for the DF. The OM models are the simplest anisotropic models.

The anisotropy profile for all OM models is universal irrespective of the density profile,

$$
\beta=\frac{r^{2}}{r^{2}+r_{\mathrm{a}}^{2}}
$$

where $r_{\mathrm{a}}$ is the model parameter. This is the only parameter that characterizes the degree of anisotropy of the models from the family in question. The velocity distribution is predominantly isotropic inside the sphere of radius $r_{\mathrm{a}}$ and significantly anisotropic outside the sphere of this radius. Note that the degree of anisotropy for all OM models tends to unity on the periphery of the system, i.e., radial orbits dominate on the periphery.

The OM models can be constructed for a density distribution corresponding to a Plummer sphere:

$$
\rho(r)=\frac{3 M}{4 \pi} \frac{a^{2}}{\left(r^{2}+a^{2}\right)^{5 / 2}},
$$

where $M$ is the total mass of the model and $a$ is the scale of the density distribution (in our virial system of units, $M=1$ and $a=3 / 16$ ). This density model was chosen as an example of a model with a nearly uniform central density distribution.

For the OM models with the density profile of a Plummer sphere, there exists a minimum value of $r_{\mathrm{a}} / a=3 / 4$ that gives a physical model with a nonnegative DF (Osipkov 1979; Merritt 1985a). The $r_{\mathrm{a}} / a$ boundary that separates the physical and nonphysical models depends on the density distribution and, in the general case (for a particular density distribution), can be found numerically (Carollo et al. 1995). However, the DF nonnegativity for the models does not yet ensure their stability. The exact stability boundary 
for the OM models can be found from numerical simulations and, below, we demonstrate some examples related to the instability of radial orbits.

We used the method described in Section 2 to construct models with the density profile (3) and the anisotropy profile (2) for several values of the parameter $r_{\mathrm{a}}$.

The parameter of the iterative method, the time of one iteration, was chosen to be $t_{i}=10$. This choice is fairly arbitrary. We can take a larger and smaller parameter $t_{i}$. The result of the iterative method is virtually independent of the chosen time of one iteration (Rodionov and Orlov 2008). The initial model for iterations was a cold model with zero velocities. Figure 1 demonstrates the convergence of iterations when a model with $r_{\mathrm{a}}=0.9$ is constructed (we will call it the POM model). We see from Fig. 1 that the profiles of the stellar velocity dispersions in the $r$ and $\varphi$ directions essentially coincide with the theoretical profiles for the corresponding OM model (the model with the density profile of a Plummer sphere and with $r_{\mathrm{a}}=0.9$ ) after 20 iterations. In general, all parameters of the POM model, to within noise, are equal to those of the OM model. We also experimentally checked that the POM model is an equilibrium one. In the course of its dynamical evolution, the POM model retains all its characteristics, to within noise, on time scales of the order of several tens of crossing times.

Below, we demonstrate the potentialities of the iterative method in constructing anisotropic models differing from the OM models. To this end, we took four anisotropy profiles that definitely do not belong to the family (2).

Figure 2 presents four profiles of the ratid $\sigma_{\varphi} / \sigma_{r}=\sqrt{1-\beta}$. We used these profiles to construct models P1, P2, P3, P4, and POM. In models P1 and P2, the $\sigma_{\varphi} / \sigma_{r}$ profile lies below and above that yielded by the OM model in the central regions and on the periphery, respectively. Thus, the fraction of radial orbits on the periphery decreased. We also considered the opposite case (models P3 and P4). For these models, the fraction of radial orbits on the periphery was higher.

Just as in the case of constructing the POM model, we took a cold model with zero velocities as the initial one. The time of one iteration was $t_{i}=10$. The iterations rapidly converged (approximately in 20 steps) for all models. This suggests that the constructed models are physical, i.e., the DF that describes the system is nonnegative. Otherwise, the iterations would not converge.

The constructed models P1, P2, and P3 turned out to be equilibrium and stable ones. In the course of their dynamical evolution, these models did not change (to within noise) on a fairly long time scale, of the order of several tens of system crossing times. They retained both the anisotropy profiles and the remaining structural and kinematic characteristics. Model P4 (with a wide isotropic core and a highly anisotropic periphery) behaved somewhat differently. This model was a dynamically equilibrium one on a short time scale, but an instability of radial orbits grew in the model on time scales of the order of several crossing times $(t=20)$. This instability manifested itself as a distortion of the initial spherically symmetric model structure on the periphery, where nearly radial orbits dominate (Fig. 3).

Models with central density peaks. The iterative method also easily constructs OM-type models for other density profiles, for example, for the Hernquist (1990) sphere,

$$
\rho(r)=\frac{M}{2 \pi} \frac{a}{r(r+a)^{3}},
$$

\footnotetext{
${ }^{2}$ In the models under consideration, $\sigma_{\varphi}=\sigma_{\theta}$.
} 
where $M$ is the total mass of the model and $a$ is the scale of the density profile (in our virial system of units, $M=1$ and $a=1 / 3$ ). The Hernquist sphere can be used to construct models that differ significantly from the OM-type models, for example, for models with predominantly radial orbits in their central regions (a high degree of anisotropy). It follows from theoretical considerations that such models are compatible only with density profiles having central peaks (see Merritt (1985a) for a discussion). The density profile (4) has such a peculiarity. In contrast, the Plummer sphere considered above is an example of a model with an almost uniform central density distribution. The central part of this model should be isotropic, since the central radial orbits are incompatible with the model having a homogeneous core.

We explored the possibilities for constructing centrally anisotropic models using the Hernquist sphere as an example, for which the anisotropy profile was specified in the form $\beta(r)=$ const. According to the theorem from An and Evans (2006), for the density profile (44) that corresponds to the Hernquist sphere, the degree of anisotropy $\beta$ in the central region cannot be higher than 0.5. For a constant anisotropy profile, $\sigma_{\varphi} / \sigma_{r}$ does not depend on the radius either. We constructed models for $\sigma_{\varphi} / \sigma_{r}=0.95,0.9,0.8$, and 0.7. We will call these models $\mathrm{HC} 0.95, \mathrm{HC} 0.9, \mathrm{HC} 0.8$, and $\mathrm{HC} 0.7$. These ratios $\sigma_{\varphi} / \sigma_{r}$ correspond to $\beta=0.0975,0.19,0.36$, and 0.51 .

For models $\mathrm{HC} 0.8$ and $\mathrm{HC} 0.7$, the iterations converge conditionally. By the twentieth iteration, the anisotropy profile did not change in the time of one iteration almost over the entire length. The innermost regions constitute an exception. The ratio $\sigma_{\varphi} / \sigma_{r}$ in the innermost regions always reached a level approximately equal to unity. Every time we obtained an isotropic core (Fig. 4) already at the next iteration step by forcibly setting a given degree of anisotropy over the entire system. Its size is slightly smaller than the core size $a$ of the Hernquist sphere, which is $1 / 3$ in the virial system of units used. This behavior of the system could be explained for model $\mathrm{HC} 0.7 \mathrm{c}$ with $\sigma_{\varphi} / \sigma_{r}=0.7$, or $\beta=0.51$. This value of $\beta$ is higher than the above critical value of $\beta=0.5$, which is compatible with the density profile of the Hernquist sphere. However, a similar behavior was also observed for model $\mathrm{HC} 0.81$ with $\sigma_{\varphi} / \sigma_{r}=0.8$. The identification of a homogeneous core probably stems from the fact that the central density peaks compatible with radial orbits are very difficult to resolve in numerical models. These difficulties are not related to the application of the potential softening procedure, since the softening length for our models $(\epsilon=0.005)$ was definitely smaller than the system core size $a=1 / 3$. The main reason why the central density peaks are poorly resolved is the finite number of particles used.

For models $\mathrm{HC} 0.95$ and $\mathrm{HC} 0.9$, the iterations converged in the sense that the anisotropy profile ceased to change and corresponded to the fixed profile (Fig. (4). However, there was a slight tendency for the core to be isotropized for these models as well.

It should be noted that the model with $\sigma_{\varphi} / \sigma_{r}=0.7$ (model HC0.7 in Fig. 4), in addition, turned out to be unstable and the initially spherical model turned into an ellipsoid in the course of its dynamical evolution.

The instability of an anisotropic Hernquist sphere was numerically considered by Buyle et al. (2006). In particular, they presented the results of their investigation of models with the anisotropy profile $\beta=\frac{\beta_{0}+\left(r / r_{\mathrm{a}}\right)^{2}}{1+\left(r / r_{\mathrm{a}}\right)^{2}}$ (Cuddeford (1991) models) for various values of $\beta_{0}$ and $r_{\mathrm{a}}$. These models are close to those we considered when $r_{\mathrm{a}} \rightarrow \infty$. As we clearly see from Fig. 5 in Buyle et al. (2007), the parameters of our model HC0.7 are outside the range of parameters for stable models. At the same time, the parameters of our remaining models ( $\mathrm{HC} 0.95, \mathrm{HC} 0.9$, and $\mathrm{HC} 0.8)$ lie in the stability region, which is also confirmed by our analysis. 
Anisotropic Dark Halos. Finally, we used our method to construct a model with a realistic anisotropy profile, the profile obtained in cosmological simulations.

We used the NFW models that well describe the density profiles of cosmological dark halos. Highresolution $N$-body simulations (Navarro et al. 1996, 1997) revealed that the halos produced by the gravitational clustering of dark matter have a universal structure. The properly scaled density distribution does not depend on the applied cosmological model. It is represented by the following simple formula:

$$
\rho(r)=\frac{4 \rho_{\mathrm{s}}}{\left(r / r_{\mathrm{s}}\right)\left(1+r / r_{\mathrm{s}}\right)^{2}},
$$

where $r_{\mathrm{S}}$ is the characteristic radius at which the logarithmic slope of the density profile is $d \ln \rho / d \ln r=-2$ and $\rho\left(r_{\mathrm{s}}\right)=\rho_{\mathrm{s}}$. The shape of the density profile is characterized by the concentration parameter $c=R_{\mathrm{vir}} / r_{\mathrm{s}}$. Here, $R_{\mathrm{vir}}$ is the so-called virial radius. It is introduced in such a way that the mean density inside the sphere of radius $R_{\text {vir }}$ is higher than the critical density specified in cosmological models by a fixed factor $\left(\delta_{\text {vir }}\right)$. In various papers, this radius is assumed to be $\Delta_{\text {vir }}=100,178,200$.

We will use a system of units with $G=1, r_{\mathrm{s}}=1$, and $r h o_{\mathrm{s}}=1 / 4$. Fixing $r_{\mathrm{s}}$ means that the virial radius can be expressed in terms of the concentration parameter. Thus, for example, the concentration parameter $c=10$ commonly used in galaxy formation models gives the virial radius $R_{\mathrm{vir}}=10$.

The density profile (5) leads to a diverging mass. Therefore, in $N$-body models, it should be cut off at some radius $R_{\text {cut }}$. To avoid the boundary effects, we assumed in our calculations that $R_{\text {cut }}=40 r_{\mathrm{s}}$. Starting from $r_{\text {cut }}=20$, we represented the density profile by a spline. At $c=10$, this gives $r_{\text {cut }}=2 R_{\text {vir }}$ and $R_{\text {cut }}=4 R_{\mathrm{vir}}$.

In $N$-body simulations, the dark matter halos have an anisotropic velocity distribution. The anisotropy profile $\beta(r)$ usually has such a structure that $\beta(r) \approx 0$ within the sphere of a radius comparable to $r_{\mathrm{s}}$. Thus, the dark halo model has an isotropic core. Further out, $\beta(r)$ gradually increases, reaching $\beta(r)=0.35-0.5$ at $r=R_{\text {vir. }}$. In terms of the ratio $\sigma_{\varphi} / \sigma_{r}$, this gives $\sigma_{\varphi} / \sigma_{r}=0.7-0.8$ (Cole and Lacey 1996; Thomas et al. 1998; Colin et al. 2000; Fukushige and Makino 2001; Diemand et al. 2004).

In our calculations, we took a $\sigma_{\varphi} / \sigma_{r}$ profile in the form

$$
\frac{\sigma_{\varphi}}{\sigma_{r}}=\frac{0.2}{\sqrt{\left(\frac{r}{0.9}\right)^{2}+1}}+0.8 .
$$

In this case, the ratio $\sigma_{\varphi} / \sigma_{r}$ is close to unity within the core of radius $r_{\mathrm{s}}=1$ and decreases to 0.8 at the distance $r=R_{\text {vir }}$.

Using the iterative method, we constructed a model with the density profile (5) and an anisotropy profile corresponding to (6). The parameter of the iterative method, the time of one iteration, was chosen to be $t_{i}=10$. The initial model for iterations was a cold model with zero velocities. As in the case of constructing the Plummer and Hernquist models described above, the iterations converged rapidly (approximately in 20 steps).

Figure 5 shows the results of our equilibrium test for the constructed model (ANFW). We see that the structural and kinematic profiles of the model are well preserved on a time scale comparable to several system crossing times.

To demonstrate the potentialities of the suggested iterative method, we constructed only one anisotropic NFW model. It turned out to be stable. However, the stability of anisotropic halo models with the density profile (5) has not been studied in the literature 
even for the simplest types of anisotropy like the OM models. Our technique allows halo models to be constructed in a broad class of anisotropy profiles. In the future, we are planning to study the stability of such models in more detail.

\section{DISCUSSION AND CONCLUSIONS}

The main advantage of the method used here is that we do not need to know an explicit expression for the DF to construct a model of the stellar system. It will suffice only to ascertain what number of integrals of motion it depends on. This determines the type of the equilibrium dynamical models being realized. Subsequently, the model dynamically adjusts itself to the specified constraints on the kinematics of the system.

Our method can be compared to the well-known method of Schwarzschild (1979) and its modification known as M2M, made-to-measure (Syer and Tremaine 1996; De Lorenzi et al. 2007). In Schwarzschild's and M2M methods, the system is constructed as a superposition of particles moving, as a rule, in a fixed potential. The weights with which the particles contribute to the density and velocity distributions can be determined by minimizing the deviations of various parameters of the model being constructed from those of the target model. Our method is simpler: the system adjusts itself to equilibrium. We only hold a number of fixed parameters. In the realization of the iterative method described here, we adjust the velocities to fixed constraints when transferring the velocity distribution from one iteration step to another.

The question arises as to how general these constraints should be and whether more detailed kinematics gives rise to systems that are actually not realizable.

For anisotropic spherically symmetric models, we can fix the velocity anisotropy profile $\beta(r)$ as a constrain on the kinematics of the system. An alternative constraint is to consider the mean degree of anisotropy of the system or the mean value of $\sigma_{\varphi} / \sigma_{r}$ for the entire system.

In this paper, we described spherically symmetric models for which the anisotropy profile was fixed from the outset. Let us make several remarks concerning the second variant of constraints.

It seemed to us that global constraints on the kinematics of the system could give some of the selected models. Thus, for example, Rodionov and Orlov (2008) used the iterative approach to construct models with a completely different geometry, stellar disks. Fixing the total angular momentum of the disk as a global parameter of the system yielded a oneparameter family of models. Their characteristic feature was a Gaussian velocity distribution in three directions. For spherically symmetric anisotropic models, all turned out to be more complex. If the mean value of $\sigma_{\varphi} / \sigma_{r}$ is fixed for the entire system, but the profile $\beta(r)$ is not maintained forcibly, then the solution is found to be ambiguous. It depends strongly on the iteration parameters (the initial velocities, the time of the system's "independent" dynamical evolution during one iteration, etc.). Thus, in the class of anisotropic models under consideration, the approach with global constraints on the kinematics of the system does not set apart any family of finite models.

Of course, our approach does not allow equilibrium anisotropic models with an arbitrary anisotropy profile to be constructed. This is possible only for $\beta(r)$ that are compatible with a nonnegative DF. If the $\operatorname{DF} f(E, L)$ corresponding to spherically symmetric anisotropic models can take on negative values at admissible $E$ and $L$, then the iterative method cannot be applied to the construction of such systems. For example, no equilibrium model of a Plummer sphere with the OM anisotropy profile (2) and $r_{a} / a<3 / 4$ can 
be constructed by the iterative method.

The constructed equilibrium models may turn out to be unstable (e.g., models P4 and HC0.7 described here). The stability criteria in stellar dynamics impose the most stringent constraints on the global parameters of real systems. In the context of the problem of constructing anisotropic spherically symmetric models, our method gives direct material for investigating the stability of such models and constraints on the possible shape of the anisotropy profile $\beta(r)$. Our method expands significantly the class of stable equilibrium models. For a given density distribution, this makes it possible to draw a boundary over the actually realizable anisotropy profiles.

The question of the stability of anisotropic models is particularly topical as applied to dark halo models with the NFW or M99 density distribution. Whereas the family of so-called $\gamma$-model 3 (see, e.g., Dehnen 1993) was analyzed for stability in sufficient detail for various anisotropy profiles (Carollo et al. 1995; Meza and Zamorano 1997; Buyle et al. 2006), the anisotropic NFW and M99 models are virtually unstudied. Since we now have a tool for constructing such models in our hands, we are planning to return to this question.

It should also be noted that the constructed anisotropic dark halo models with a density distribution corresponding to cosmological dark halos can serve as direct input data for investigating the dynamics of such systems in $N$-body simulations. It is easy to generalize the models described here to spherically symmetric anisotropic models with rotation. These models can be used in the problems of gas accretion onto dark halos and in modeling the formation of disk galaxies. Comparison of the global parameters for stellar disks at various stages of their formation with observational data for disk galaxies at various redshifts can give an estimate of the relative efficiency of the external and internal processes that affect the evolution of galaxies. Another result of solving this problem can be the verification of several relations between structural and kinematic parameters of the disk and the halo that follow from the well-known semi-analytical models for the formation of disk galaxies (White and Rees 1978; Fall and Efstathiou 1980; Blumenthal et al. 1986; Mo et al. 1998).

\section{ACKNOWLEDGMENTS}

This work was supported by the Russian Foundation for Basic Research (project no. 06-02-016459) and a grant for support of leading scientific schools from the President of Russia (NSh-8542.2006.2).

\section{REFERENCES}

1. J. H. An and N. W. Evans, Astron. J. 131, 782 (2006).

2. E. Athanassoula, Mon. Not. R. Astron. Soc. 341, 1179 (2003).

3. M. Baes and E. Van Hese, Astron. Astrophys. 471, 419 (2007).

4. J. Barnes, Astrophys. J. 331, 699 (1988).

5. J. Barnes and P. Hut, Nature 324, 446 (1986).

6. J. Binney and S. Tremaine, Galactic Dynamics (Princeton Univ. Press, Princeton, 1987).

7. G. R. Blumenthal, S. M. Faber, R. Flores, and J. R. Primack, Astrophys. J. 301, 27 (1986).

\footnotetext{
${ }^{3}$ The Hernquist sphere belongs to this family.
} 
8. P. Buyle, E. Van Hese, S. De Rijcke, and H. Dejonghe, Mon. Not. R. Astron. Soc. 375, 1157 (2006).

9. C. M. Carollo, P. T. de Zeeuw, and R. P. van der Marel, Mon. Not. R. Astron. Soc. 276, 1131 (1995).

10. Sh. Cole and C. Lacey, Mon. Not. R. Astron. Soc. 281, 716 (1996).

11. P. Coli n, A. A. Klypin, and A. V. Kravtsov, Astrophys. J. 539, 561 (2000).

12. P. Cuddeford, Mon. Not. R. Astron. Soc. 253, 414 (1991).

13. A. Curir, P. Mazzei, and G. Murante, Astron. Astrophys. 467, 509 (2007).

14. F. De Lorenzi, V. P. Debattista, O. Gerhard, and N. Sambhus, Mon. Not. R. Astron. Soc. 376,71 (2007).

15. W. Dehnen, Mon. Not. R. Astron. Soc. 265, 250 (1993).

16. J. Diemand, B. Moore, and J. Stadel, Mon. Not. R. Astron. Soc. 352, 535 (2004).

17. G. Efstathiou and B. J. T. Jones, Mon. Not. R. Astron. Soc. 186, 133 (1979).

18. S. M. Fall and G. Efstathiou, Mon. Not. R. Astron. Soc. 193, 189 (1980).

19. N. Fukushige and J. Makino, Astrophys. J. 557, 533 (2001).

20. L. Hernquist, Astrophys. J. 356, 359 (1990).

21. L. Hernquist, Astron. Astrophys. Suppl. Ser. 86, 389 (1993).

22. A. Klypin, A. Kravtsov, J. Bullock, and J. Primack, Astrophys. J. 554, 903 (2001).

23. E. L. Lokas and G. A. Mammon, Mon. Not. R. Astron. Soc. 321, 155 (2001).

24. A. Meza and N. Zamorano, Astrophys. J. 490, 136 (1997).

25. D. Merritt, Astron. J. 90, 1027 (1985a).

26. D. Merritt, Mon. Not. R. Astron. Soc. 214, 25 (1985b).

27. H. J. Mo, S. Mao, and S. D. M. White, Mon. Not. R. Astron. Soc. 295, 319 (1998).

28. B. Moore, T. Quinn, F. Governato, J. Stadel, and G. Lake, Mon. Not. R. Astron. Soc. 310, 1147 (1999).

29. J. F. Navarro, C. S. Frenk, and S. D. M. White, Astrophys. J. 462, 563 (1996).

30. J. F. Navarro, C. S. Frenk, and S. D. M. White, Astrophys. J. 490, 493 (1997).

31. L. P. Osipkov, Pis'ma Astron. Zh. 5, 77 (1979) [Sov. Astron. Lett. 5, 42 (1979)].

32. J. P. Ostriker and P. J. E. Peebles, Astrophys. J. 186, 467 (1973).

33. S. A. Rodionov and V. V. Orlov, Mon. Not. R. Astron. Soc. 385, 200 (2008).

34. S. A. Rodionov and N. Ya. Sotnikova, Astron. Zh. 82, 527 (2005) [Astron. Rep. 49, $470(2005)]$.

35. S. A. Rodionov and N. Ya. Sotnikova, Astron. Zh. 83, 1091 (2006) [Astron. Rep. 50, 983 (2006)].

36. M. Schwarzschild, Astrophys. J. 232, 236 (1979).

37. M. Schwarzschild, Astrophys. J. 409, 563 (1993).

38. D. Syer and S. Tremaine, Mon. Not. R. Astron. Soc. 282, 223 (1996).

39. P. J. Teuben, ASP Conf. Ser. 77, 398 (1995).

40. P. A. Thomas, J. M. Colberg, H. M. P. Couchman, et al., Mon. Not. R. Astron. Soc. 296, 1061 (1998).

41. I. R. Walker, C. Mihos, and L. Hernquist, Astrophys. J. 460, 121 (1996).

42. S. D. M. White and M. J. Rees, Mon. Not. R. Astron. Soc. 183, 341 (1978).

43. L. M. Widrow, Astrophys. J. Suppl. Ser. 131, 39 (2000).

Translated by V. Astakhov 

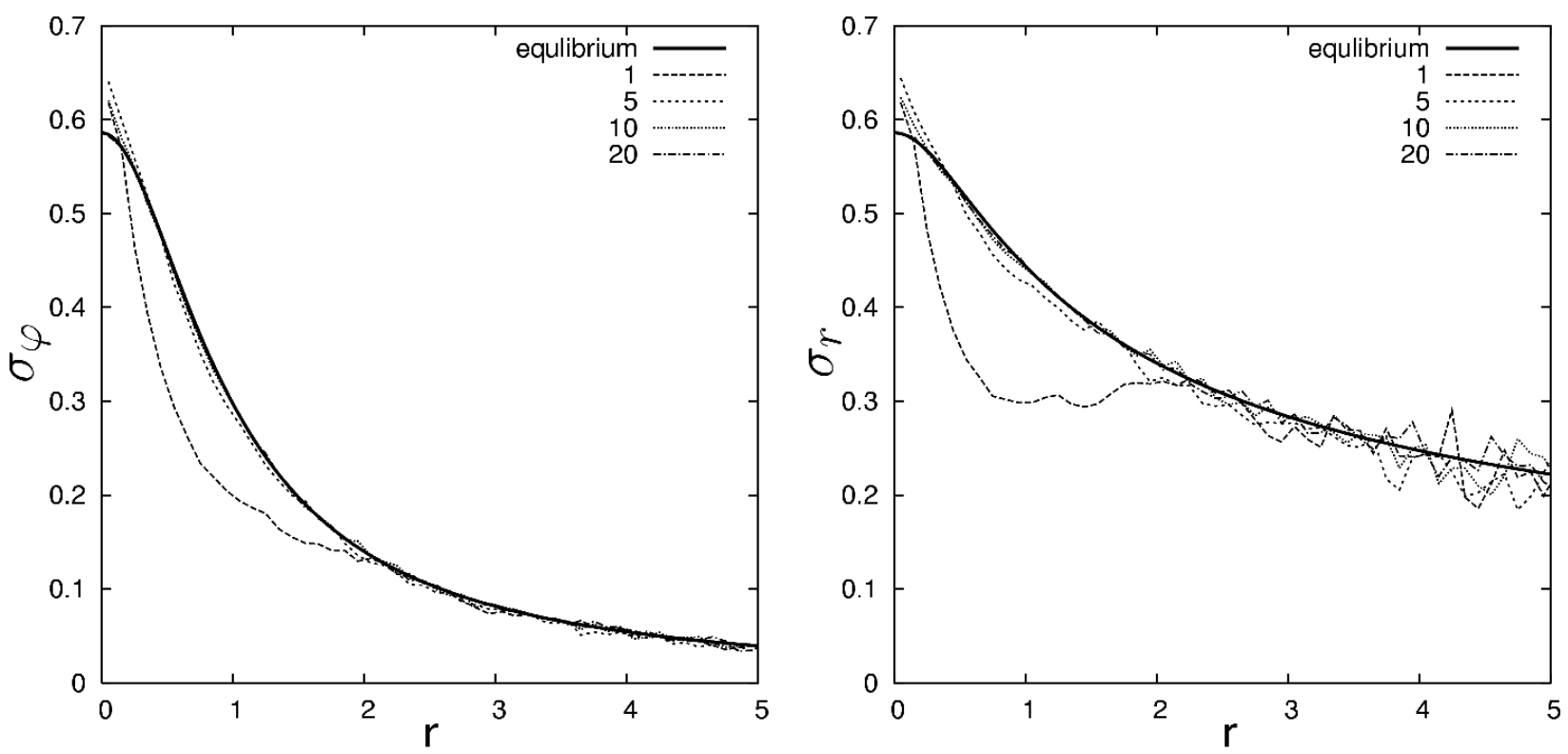

Fig. 1: Convergence of iterations when a Plummer sphere with an anisotropy profile corresponding to the OM model with the parameter $r_{a}=0.9$ (the POM model) is constructed: (a) the $r$ dependence of the azimuthal velocity dispersion $\sigma_{\varphi}$; (b) the $r$ dependence of the radial velocity dispersion $\sigma_{r}$. The profiles are presented for 1, 5, 10, and 20 iterations. The thick solid line indicates the theoretical values of $\sigma_{\varphi}$ and $\sigma_{r}$ for the OM model (Merritt 1985a). Note that the profiles for iteration 20 are virtually identical to the theoretical ones. 




Fig. 2: Radial profiles of the ratio $\sigma_{\varphi} / \sigma_{r}$ that were used to construct models P1, P2, P3, and P4 differing from the POM model. The profile of the POM model corresponds to the OM model with $r_{a}=0.9$. 



Fig. 3: Representation of model P4 in the $x-y$ plane at (a) $t=0$ and (b) $t=80$. The shades of gray show the logarithm of the number of particles per pixel. The instability of radial orbits in the outer part of the model manifests itself as a distortion of the initial spherically symmetric model structure.
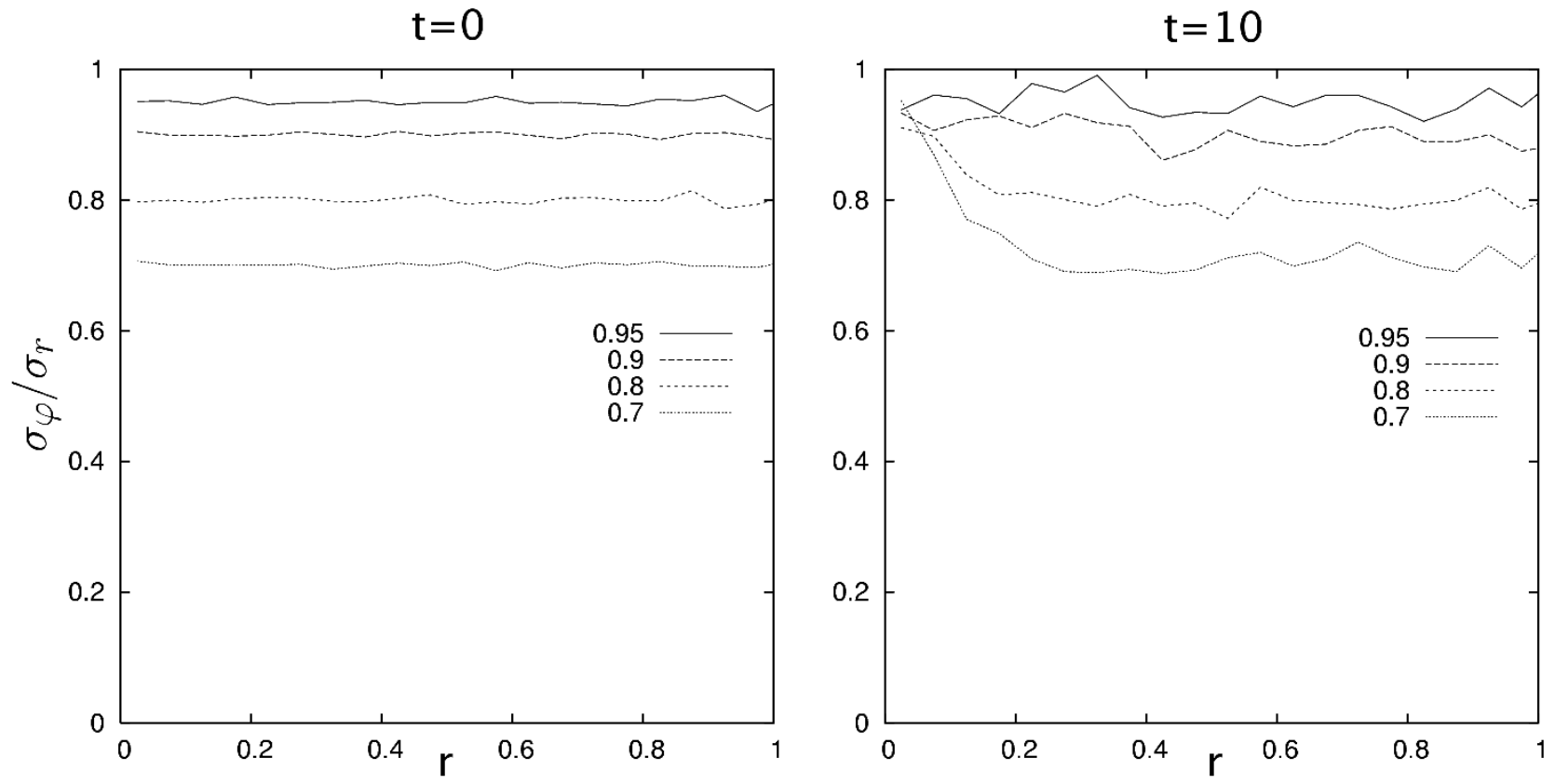

Fig. 4: Ratio $\sigma_{\varphi} / \sigma_{r}$ versus $r$ for models $\mathrm{HC} 0.95, \mathrm{HC} 0.9$, HC0.8, and HC0.7 for two times, (a) $t=0$ and (b) $t=10$. 

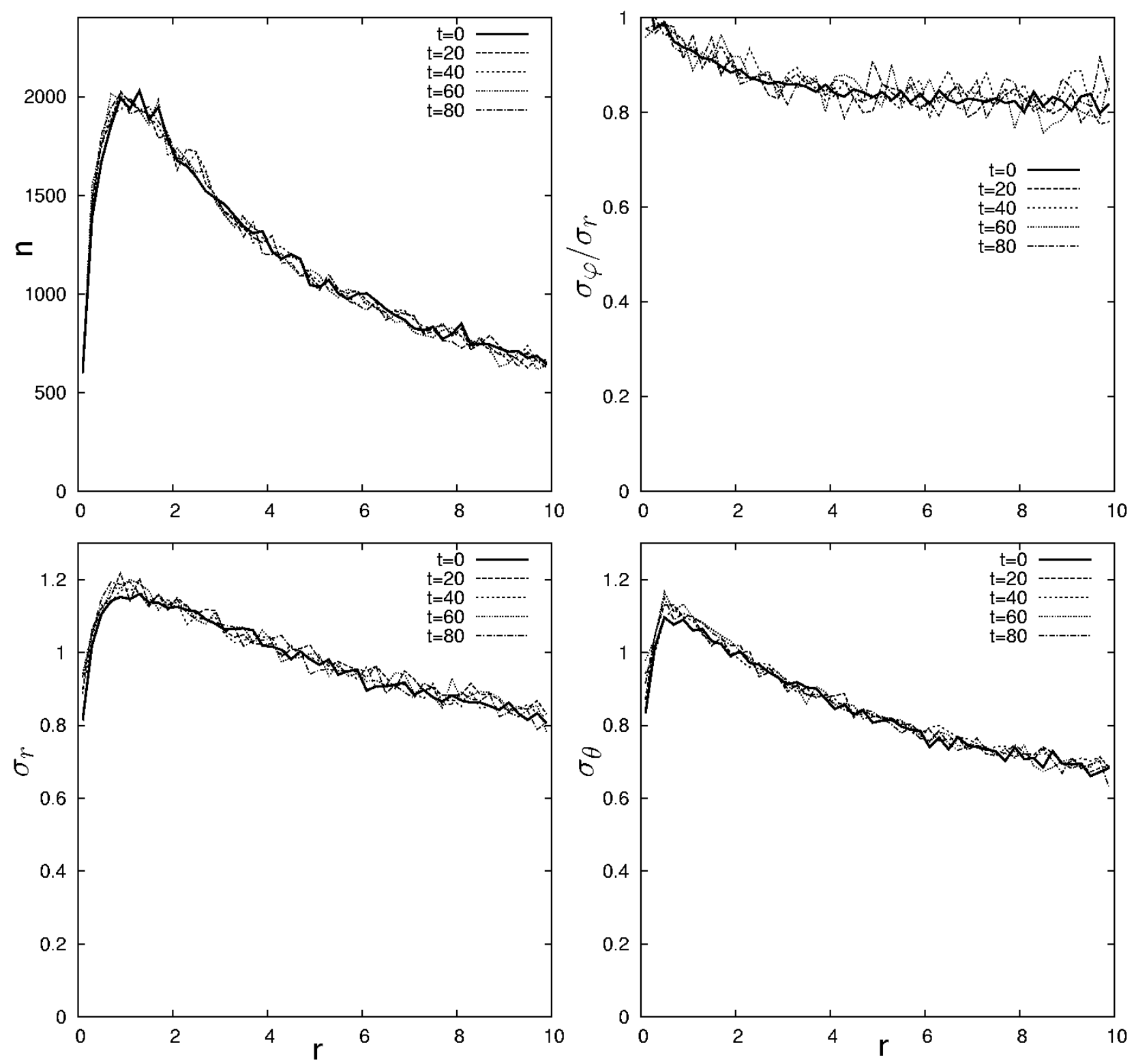

Fig. 5: Equilibrium test for the ANFW model. For several times $(t=0,20,40,60$, and 80), the radial profiles are presented for the following quantities: $n$ is the number of particles in concentric spherical layers, $\sigma_{\varphi} / \sigma_{r}, \sigma_{r}$ and $\sigma_{\theta}$. 